\title{
Familiarity of Actors Affects Eye Gaze Processing During Observation of Goal-Directed Actions
}

\author{
Mitsuhiko Ishikawa* and Shoji Itakura \\ Department of Psychology, Kyoto University, Japan
}

Submission: March 09, 2017; Published: March 22, 2017

*Corresponding author: Mitsuhiko Ishikawa, Department of Psychology, Graduate School of Letters, Kyoto University, Yoshida-honmachi, Sakyo, Kyoto 606-8501, Japan, Tel: +81 (0) 75753 2693; Email: ishikawa.mitsuhiko.23r@st.kyoto-u.ac.jp

\begin{abstract}
Eye gaze is one of the most important signals of others' mental states, and the Mirror Neuron System (MNS) has been proposed as the neural basis of mind-reading. Both eye gaze processing and MNS activation are apparently facilitated by familiarity. We examined how the familiarity of actors affects the eye gaze process during observation of goal-directed actions. Fifteen pairs of acquaintances participated; one member of each pair acted in the stimulus video, and the other performed an eye tracking task and a Posner task cued by eye gaze. Stimuli for the eye tracking task comprised goal-directed and non-goal-directed actions. Familiarity increased reference to the face as participants watched goal-directed actions. This finding may be related to familiarity effects on social motivation, especially with respect to mind-reading.
\end{abstract}

Keywords: Familiarity; Eye gaze; Goal-directed action; Posner cuing task; Mirror Neuron System

Abbreviations: fMRI: functional Magnetic Resonance Imaging; MNS: Mirror Neuron System; IFG: Inferior Frontal Gyrus; IPL: Inferior Parietal Lobule; SOA: Stimulus Onset Asynchrony

\section{Introduction}

Eye gaze is one of the most important signals for nonverbal communication, especially in attribution of the mental states of others, such as their intention or desires [1,2]. It has been said that humans use eye gaze for "mind-reading" [3]. Recently, many studies on the neural basis of mind-reading have been conducted, often using functional magnetic resonance imaging (fMRI). The Mirror Neuron System (MNS), composed of motor-related brain regions in the inferior frontal gyrus (IFG) and inferior parietal lobule (IPL), has been regarded as the neural network behind mental attributions, especially the understanding of the intentions of actions [4].

This understanding may be modulated by familiarity. The activities in these brain regions during intention understanding have been shown to be affected by physical characteristics of the actors (e.g., their race [5]). Individuals physically similar to oneself may be more readily mapped onto sensorimotor representations of the self, and the process of perceptual familiarity may affect the activity of the MNS. In children, significantly greater MNS activity has been shown with familiar actors (caregivers or siblings) than with strangers [6]. Eye tracking techniques have also been used to examine action intentions. Proactive goal-directed eye movements have been observed in adults as they watched goal-directed actions [7]. Predictive eye gaze may be modulated by the state of the motor system [8].

Many studies that have examined predictive eye gaze cues during observation of goal-directed actions have failed to consider possible effects of the actors' faces. Myowa-Yamakoshi et al. [9] compared eye gaze of humans and chimpanzees as they watched goal-directed actions in which the actors' faces were shown. The results revealed that humans looked longer at the face area than did chimpanzees as the actor approached her action goal. This result suggests that humans may refer to faces to confirm eye gaze direction, and reference to faces can be used to understand action goals and intentions.

0'Toole et al. [10] have suggested that the recognition of social communication information from dynamic human faces, such as eye gaze and expression, may be mediated by familiarity. Facial familiarity and self-similarity have been shown to enhance gaze cuing effects in adults $[11,12]$. Thus dynamic aspects of familiar faces, including gaze direction and head orientation, may promote the understanding of action intentions. Here, we used an eye tracking technique to test the effects of familiarity on eye gaze processing during observation of goal-directed 
actions. We predicted that familiarity would modulate fixations to the actor's face area, such that eye gaze direction could be used to understand action goals.

\section{Materials and Method}

\section{Participants}

Fifteen pairs of adults (16 males, 14 females; mean age $19.93 \pm 1.8$ years) who knew each other were recruited. Behavioral data were collected from the other 15 participants (8 males, 7 females). All 30 participants were asked to rate how often they had previously seen the actors on a 1-5 scale ( 1 = never; 5 = very often). This rating was used to distinguish Friend and Stranger conditions. All participants in the Friend condition were evaluated has having been seen often. The study was carried out in accordance with the provisions of the World Medical Association Declaration of Helsinki, and informed written consent was obtained from each participant. The protocol was approved by the Research Ethics Review Board at the Department of Psychology, Kyoto University, Kyoto, Japan.

\section{Apparatus and stimuli}

A Tobii (Stockholm, Sweden) T60 Eye Tracker with a 17 inch TFT monitor was used to present videos and direct-gaze pictures and to record eye movements using a $60 \mathrm{~Hz}$ sampling rate (Tobii Studio 2.2.8, Tobii Technology). Participants were seated approximately $60 \mathrm{~cm}$ from the monitor. The video stimuli and areas of interest (AOIs) for analysis are illustrated in Figure 1. In a Posner cuing task [13], stimuli were presented on a laptop (NEC LavieG) and participants responded via a keyboard (Figure 1).

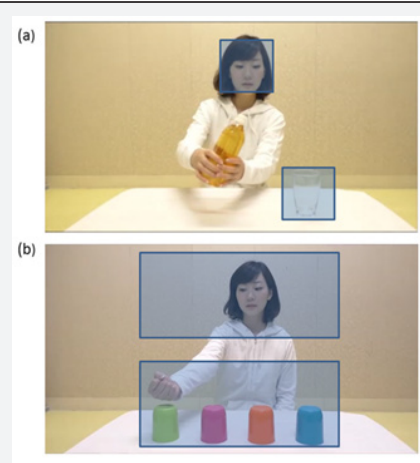

Figure 1: Example of a scene from the video stimulus used in each condition, showing areas of interest (AOls; blue shaded boxes) used for analysis. (a) Goal-directed action, pouring tea into a glass. (b) Non-goal-directed action, moving the backs of the hands over four cups.

One member of each pair was selected to generate the stimuli for the Friend condition. Videos of Goal-directed and Non-goaldirected actions were constructed. In the Goal-directed condition (Figure 1a), the actors were told to hold a plastic bottle above the table with both hands for 4 seconds, then to move the plastic bottle to the glass within 2 seconds, to pour tea until the glass was 70 percent filled within 6 seconds, and finally to return to the first position and remain there for 4 seconds. In the Nongoal-directed condition (Figure $1 \mathrm{~b}$ ), the actors were instructed to move the back of one hand over one of four cups for 2 seconds, and return the hand to the starting position within 2 seconds. The actor repeated this action for all four cups using alternate hands. All videos were 16 seconds in duration. The Direct gaze pictures ( 50 wide $\times 7 \mathrm{o}$ high) were presented 5 seconds in black and white. For the Posner task cued by eye gaze [14], three faces were presented in black and white, each 50 wide $\times 7$ o high: a face with eyes closed for a pre-cuing stimulus and right- and left-gazing faces. The Stranger condition was also comprised the same components, but actors were chosen from students who had never seen by participants.

\section{Procedure}

The order of presentation of the eye tracking task and the Posner task was counterbalanced between participants. In the eye tracking task, after an initial calibration procedure, each participant completed two trials in each of the conditions (Friend condition; Stranger condition) for 3 different stimuli (Direct gaze; Goal-directed action; Non-goal-directed action), presented in a pseudo-random order. The Posner task comprised 96 trials. On each trial, a fixation cross (10) was centrally displayed ( $675 \mathrm{~ms}$ ) and then an eye-closed face (5o wide $\times 7 \mathrm{o}$ high) was presented $(900 \mathrm{~ms})$, followed by a face gazing either right or left for either 100, 300, or 700 ms (Stimulus Onset Asynchrony, SOA), selected randomly with equal probability. A target asterisk (10) then appeared positioned $10 \mathrm{o}$ to the left or right of the fixation cross. Participants were required to press, as soon as possible, the ' $\mathrm{Z}$ ' key when the target appeared on the left and the ' $\mathrm{M}$ ' key when the target appeared on the right. It was emphasized that gaze direction was not relevant to target position.

\section{Results}

We defined AOIs of the same size for each condition: in the Goal-directed condition, AOIs covering the glass (Glass AOI) and the face (Face AOI); in the Non-goal-directed condition, AOIs covering the cups (Object AOI) and the actor's face (Face AOI); and in the Direct-gaze condition, AOIs covering the eyes and mouth. All eye tracking data were analysed as proportions of looking time.

In the analysis of the Goal-directed action, $2 \times 2$ ANOVAs with two levels of familiarity (Friend, Stranger) and two levels of AOI (Face, Glass) were conducted for the 2 phases (before goal; before the plastic bottle approached to the Glass AOI, after goal; after the actor started pouring tea) respectively. For the before-goal phase, a significant interaction between familiarity and AOI was observed, $\mathrm{F}(1,14)=10.730, \mathrm{p}=.006, \eta \mathrm{p} 2=.434$, with the duration of the Friend condition longer than that of the Stranger condition for the Face area, $\mathrm{p}=.012, \eta \mathrm{p} 2=.370$ (Figure 2). No significant difference was observed between Friend and Stranger conditions for predictive eye gaze at the Glass area, $\mathrm{p}=.169, \eta \mathrm{p} 2=.131$. A significant main effect of AOI revealed a 
longer fixation duration at the Face area than at the Glass area, $\mathrm{F}$ $(1,14)=38.547, \mathrm{p}<.001, \eta \mathrm{p} 2=.734$ (Figure 2).

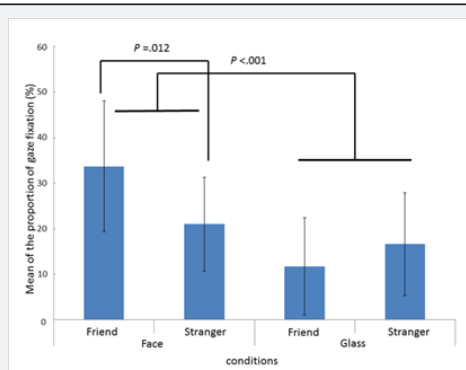

Figure 2: Mean fixation durations for four conditions in the before-goal phase.

The analysis of the after-goal phase revealed only a significant main effect of $\mathrm{AOI}(\mathrm{F}(1,14)=11.764, \mathrm{p}=.004, \eta \mathrm{p} 2=$ .457), with the fixation duration at the Glass area longer than that at the Face area. In the analysis of the Non-goal-directed action, only a significant main effect of AOI was obtained, F $(1,14)=12.287, p=.003, \eta p 2=.467$, with longer fixations at the Object area than at the Face area. The main effect of AOI was also significant in the analysis of Direct gaze, $F(1,14)=11.764, \mathrm{p}=$ $.004, \eta p 2=.457$, with longer fixations at the Eye area than at the Mouth area. For the Posner task, the cue effect was defined as mean response time in the cue congruent condition subtracted from that in the incongruent conditions. A $2 \times 3$ ANOVA with two levels of familiarity (Friend, Stranger) and three levels of SOA (100 ms, $300 \mathrm{~ms}, 700 \mathrm{~ms}$ ) revealed only a significant effect of SOA, $F(2,28)=7.484, p=.002, \eta p 2=.348$, with the $100 \mathrm{~ms}$ SOA producing a greater cue effect than the $700 \mathrm{~ms}$ SOA, $\mathrm{p}=.008$, $\eta \mathrm{p} 2=.489$.

\section{Discussion}

This study examined the effect of familiarity on eye gaze processing. By using pairs of participants known to each other, we discovered that familiarity modulated fixations to the face area before the actors approached their action goals. There was no effect of familiarity on Non-goal-directed actions, direct gaze, or the Posner task.

Myowa-Yamakoshi et al. [9] suggested that attention to faces, which potentially includes referential information such as gaze direction or emotional expression toward the target object, may be involved in the coding of goal-directed actions. Head orientation may be another important clue for gaze direction in the understanding of action goals. Our results suggest that familiarity may facilitate the motivation to understand action intentions that are goal-directed.

Some studies have examined the effects of familiarity on the eye gaze process $[15,16]$, however, most defined familiarity in terms of the in-group, for instance, sameness of race or political group. When Deaner et al. [11] defined familiarity as people who were in the same department of a university, performance on a
Posner task revealed that familiarity promoted gaze cuing only in females. We did not observe a significant effect of familiarity in our Posner task, but our sample size was limited, because our main purpose was to examine the effect of familiarity on understanding action intentions. Furthermore, the results of a Posner task do not reflect the use of eye gaze in understanding others' mental states, but rather its use in autonomic target following [17]. Hence, it is possible that familiarity only affects eye gaze processing related to mind-reading.

Although our results did not indicate that predictive eye gaze was enhanced by familiarity in the before-goal phase, this was observed in both familiar and stranger conditions with goaldirected actions. This result could be attributable to a ceiling effect, because the action goal used in this experiment was simple for adults. However, the direction of eye gaze may provide an essential clue for inferring mental states in social situations, especially for understanding action goals.

\section{Conclusion}

Our findings suggest that familiarity affects eye gaze processing related to an action goal, and studies of social cognition that manipulate personal familiarity should add to our knowledge of social behavior in real situations

\section{References}

1. Senju A, Johnson MH (2009) Atypical eye contact in autism: Models, mechanisms and development. Neurosci Biobehav Rev 33(8): 12041214.

2. Gobel MS, Kim HS, Richardson DC (2015) The dual function of social gaze. Cognition 136: 359-364.

3. Baron-Cohen S (1994) How to build a baby that can read minds: Cognitive mechanisms in mindreading. Cahiers de Psychologie Cognitive/Current Psychology of Cognition 13(5): 513-552.

4. Iacoboni M, Molnar-Szakacs I, Gallese V, Buccino G, Mazziotta JC, et al. (2005) Grasping the intentions of others with one's own mirror neuron system. PLoS Biol 3(3): e79.

5. Liew SL, Han S, Aziz-Zadeh L (2011) Familiarity modulates mirror neuron and mentalizing regions during intention understanding. Hum Brain Mapp 32(11): 1986-1997.

6. Oberman LM, Ramachandran VS, Pineda JA (2008) Modulation of mu suppression in children with autism spectrum disorders in response to familiar or unfamiliar stimuli: The mirror neuron hypothesis. Neuropsychologia 46(5): 1558-1565.

7. Flanagan JR, Johansson RS (2003) Action plans used in action observation. Nature 424: 769-771.

8. Ambrosini E, Sinigaglia C, Costantini M (2012) Tie my hands, tie my eyes. J Exp Psychol Hum Percept Perform 38(2): 263-266.

9. Myowa-Yamakoshi M, Scola C, Hirata S (2012) Humans and chimpanzees attend differently to goal-directed actions. Nature Communications 3: 693.

10. O’Toole AJ, Roark DA, Abdi H (2002) Recognizing moving faces: A psychological and neural synthesis. Trends in Cognitive Sciences 6(6): 261-266.

11. Deaner RO, Shepherd SV, Platt ML (2007) Familiarity accentuates gaze cuing in women but not men. Biology Letters 3(1): 65-68. 
12. Hungr CJ, Hunt AR (2012) Physical self-similarity enhances the gazecueing effect. Q J Exp Psychol (Hove) 65(7): 1250-1259.

13. Posner MI (1980) Orienting of attention. Q J Exp Psychol 32(1): 3-25.

14. Senju A, Tojo Y, Dairoku H, Hasegawa T (2004) Reflexive orienting in response to eye gaze and an arrow in children with and without autism. J Child Psychol Psychiatry 45(3): 445-458.

15. Pavan G, Dalmaso M, Galfano G, Castelli L (2011) Racial group membership is associated to gaze-mediated orienting in Italy. PLoS One 6(10): e25608.
16. Liuzza MT, Cazzato V, Vecchione M, Crostella F, Caprara GV, et al. (2011) Follow my eyes: The gaze of politicians reflexively captures the gaze of ingroup voters. PLoS One 6(9): e25117.

17. Teufel C, Alexis DM, Clayton NS, Davis G (2010) Mental-state attribution drives rapid, reflexive gaze following. Atten Percept Psychophys 72(3): 695-705.
Your next submission with Juniper Publishers will reach you the below assets

- Quality Editorial service

- Swift Peer Review

- Reprints availability

- E-prints Service

- Manuscript Podcast for convenient understanding

- Global attainment for your research

- Manuscript accessibility in different formats ( Pdf, E-pub, Full Text, Audio)

- Unceasing customer service

Track the below URL for one-step submission https://juniperpublishers.com/online-submission.php 